\title{
Aprendizaje colaborativo y su influencia en el rendimiento académico de los estudiantes de la Facultad de Ciencias Sociales, Universidad Nacional José Faustino Sánchez Carrión - Huacho
}

\section{Collaborative learning and its influence on the academic performance of students at the Faculty of Social Sciences, Jose Faustino Sánchez Carrion National University - Huacho}

\author{
Recibido: 12/10/2015 Revisado: 28/10/2015 Aceptado: 26/11/2015
}

Celedonio Díaz, Isabel Elcira', Pesantes Calderón, Gilberth²

\begin{abstract}
RESUMEN
Objetivos: Determinar y explicar la influencia del aprendizaje colaborativo en el rendimiento académico de los estudiantes de la Facultad de Ciencias Sociales de la Universidad Nacional José Faustino Sánchez Carrión Huacho 2013. Material y métodos: La población de estudio estuvo constituido por 1139 estudiantes matriculados, obteniéndose una muestra de 287 alumnos. Para el recojo de la información se aplicó un cuestionario a los estudiantes de las tres escuelas: Ciencias de la Comunicación se encuesto a 112 alumnos, 80 de la Escuela de Sociología y 95 de Trabajo Social. Resultados: El conocimiento y aplicación de estrategias de aprendizaje colaborativo de los estudiantes en sus materias influye significativamente en el rendimiento académico obteniendo la mejora en el mismo: Ciencias de la Comunicación $(79 \%)$, Sociología $(80 \%)$ y Trabajo Social (78\%). Conclusión: Se ha logrado revertir los resultados deficientes encontrados en el pre-test, obteniendo en el post-test un incremento significativo en la influencia del rendimiento académico de los estudiantes de la Facultad de Ciencias Sociales
\end{abstract}

Palabras clave: Aprendizaje colaborativo, aprendizaje grupal, métodos participativos.

\section{ABSTRACT}

Objective: To identify and explain the influence of collaborative learning in the academic performance of students at the Faculty of Social Sciences José Faustino Sánchez Carrión-Huacho 2013 National University. Material and methods. The study population consisted of 1139 students enrolled, obtaining a sample of 287 students. To gather the information, a questionnaire was administered to students in three schools: Communication Sciences 112 students, 80 from the School of Sociology and Social Work 95 were surveyed. Results: Knowledge and application of collaborative learning strategies of students in their subjects significantly influence academic performance improvement obtained in the same: Communication Sciences (79\%), Sociology (80\%) and social work (78\%). Conclusion: It has managed to reverse the poor results found in the pre-test, getting in the post-test a significant increase in the influence of the academic performance of students of the Faculty of Social Sciences.
Keywords: Collaborative learning, group learning, participatory methods.

\section{INTRODUCCIÓN}

Las investigaciones y estudios previos, sostienen que el aprendizaje individual y grupal con la aplicación del aprendizaje colaborativo como estrategia pedagógica docente desarrolla una buena interacción entre estudiantes y docentes; del mismo modo se pudo comprobar que el nivel de aprendizaje en los estudiantes es mejorado y su rendimiento académico también (Torres Chacón, 1998).

El aprendizaje colaborativo es un elemento al que forzosamente tenemos que referirnos cuando hablamos de innovación educativa. Su inserción en el campo educativo ha generado una revolución que mueve el corazón de la práctica docente, la cual hasta hace muy poco había sido dominada por esquemas individuales y de competencia que limitaban los procesos de aprendizaje.

La investigación educativa no sólo ha mostrado el éxito de las prácticas colaborativas en el desarrollo de los estudiantes, sino que ha enfatizado la importancia del desarrollo de habilidades colaborativas como un elemento deseable tanto en la sociedad en la que vivimos como en la sociedad del futuro.(Johnson y Halubec, 1995).

El aprendizaje colaborativo es la base para cualquier técnica que implique trabajo en equipo; va más allá del beneficio individual, ya que no sólo potencia el aprendizaje individual, sino que busca el beneficio del grupo.

En el aprendizaje colaborativo se resalta la importancia de alcanzar objetivos comunes vs. Objetivos individuales o de competencia, por lo que ayuda a lograr paralelamente objetivos de aprendizaje y objetivos de habilidades sociales y colaborativas

tales como: cooperación, tolerancia, comunicación, escucha activa y compromiso. El trabajo se realiza en grupos pequeños, generalmente heterogéneos, de tres a cuatro integrantes.

Dos de los principales representantes en la puesta en marcha de experiencias exitosas en el aprendizaje colaborativo son los hermanos Roger y David Johnson, quienes durante más de tres décadas se han dedicado a la investigación e implementación de actividades colaborativas desde niveles básicos hasta educación superior. Ellos sostienen que el trabajo en equipo permite

Facultad de Ciencias Sociales. Universidad Nacional José Faustino Sánchez Carrión. Email: iecd_24@hotmail.com

Facultad de Ciencias. Universidad Nacional José Faustino Sánchez Carrión. 
desarrollar el aprendizaje, mejorar el rendimiento académico, habilidades sociales y colaborativas como: la tolerancia, comunicación y compromiso con el trabajo en grupo; es de esta forma que el aprendizaje colaborativo brinda resultados óptimos en el proceso de enseñanzaaprendizaje de los estudiantes porque, además de lo mencionado, los prepara para el trabajo profesional, con la colaboración de cada uno, se logra alcanzar las metas y objetivos de todos.

Por todo ello, el objetivo de la investigación; es determinar y explicar la influencia del aprendizaje colaborativo en el rendimiento académico de los estudiantes de la Facultad de Ciencias Sociales de la Universidad Nacional José Faustino Sánchez Carrión Huacho 2013.

\section{MATERIAL Y MÉTODOS}

El presente trabajo de investigación se realizó en la Facultad de Ciencias Sociales en sus tres escuelas académicas (Ciencias de la Comunicación, Sociología y Trabajo Social) de la Universidad Nacional José Faustino Sánchez Carrión (UNJFSC). Siendo un estudio de nivel descriptivo explicativo donde se empleó el método científico deductivo inductivo con un diseño cuasi-experimental (pretest y un post-test), y según el tiempo de tipo transversal prospectivo.

La población de estudio constituido por 1139 estudiantes de la Facultad de Ciencias Sociales distribuidos en sus tres escuelas: 287 en Ciencias de la Comunicación, 220 en Sociología y 257 en Trabajo Social con una muestra 287 estudiantes para toda la facultad de ciencias sociales y donde la escuela de ciencias de la comunicación serán encuestados 112 alumnos, 80 de la escuela de sociología y 95 de trabajo social.

Para el recojo de los saberes previos de los estudiantes sobre la aplicación de estrategias de aprendizaje pre-test y post-test se aplicó un cuestionario con 30 ítems.

\section{RESULTADOS}

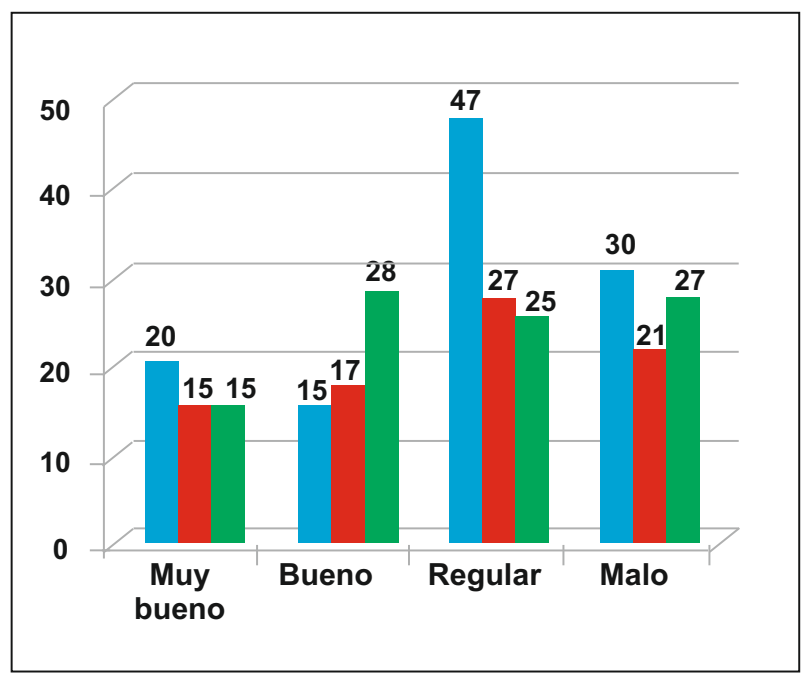

Figura 1. Distribución de los estudiantes de la Facultad de Ciencias Sociales según el rendimiento académico a partir de la influencia Significativa del aprendizaje colaborativo aplicado. (Pre-test).

Tabla 2. Distribución de los estudiantes de la Facultad de Ciencias Sociales según la influencia significativa en el rendimiento académico de los estudiantes.

\begin{tabular}{lcccc}
\hline \multirow{2}{*}{ Escuela } & \multicolumn{4}{c}{ Rendimiento Académico } \\
\cline { 2 - 5 } & Muy bueno & Bueno & Regular & Malo \\
\hline Ciencias de la Comunicación & 45 & 42 & 20 & 5 \\
Sociología & 30 & 33 & 11 & 6 \\
Trabajo Social & 37 & 36 & 17 & 5 \\
\hline
\end{tabular}

Fuente: Cuestionario desarrollado.

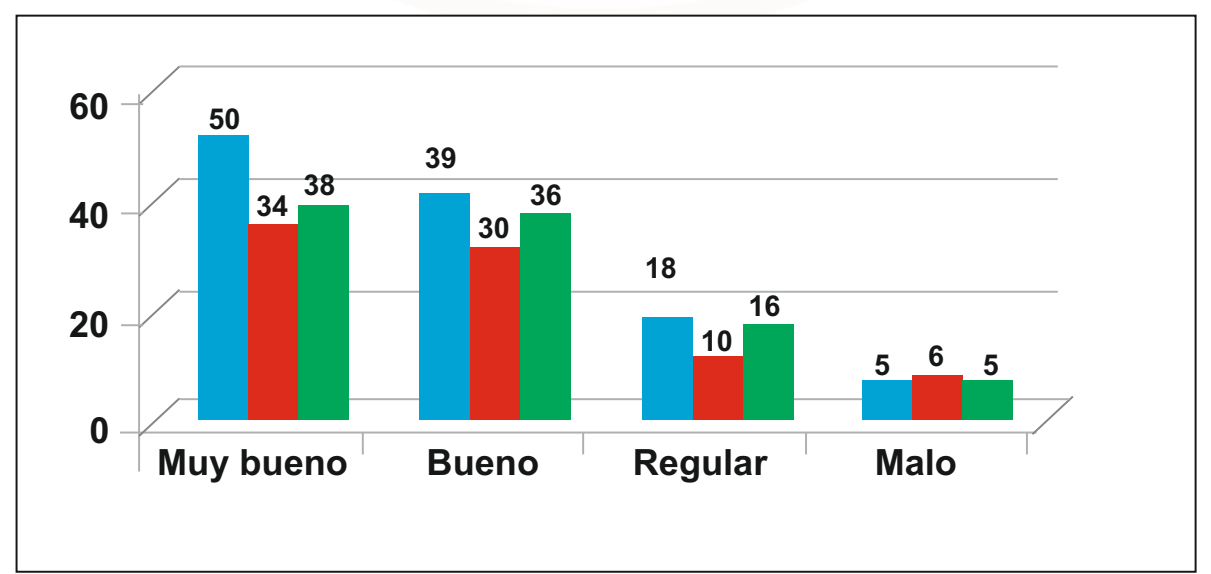

Figura 3. Distribución de los estudiantes de la Facultad de Ciencias Sociales según el efecto de la aplicación del aprendizaje colaborativo y determinar su influencia significativa en el rendimiento académico de los estudiantes. 


\section{DISCUSIÓN}

El aprendizaje individual y grupal con la aplicación del aprendizaje colaborativo como estrategia pedagógica docente desarrolla una buena interacción entre estudiantes y docentes; del mismo modo se pudo comprobar que el nivel de aprendizaje en los estudiantes es mejorado y su rendimiento académico también.

Con la aplicación del presente trabajo de investigación se ha logrado revertir los resultados deficientes encontrados en el pre-test, obteniendo en el post-test un incremento significativo en la influencia del rendimiento académico de los estudiantes de la Facultad de Ciencias Sociales; que se muestra en la tabla 3 y la figura 3 donde se aprecia los siguientes porcentajes: ciencias de la comunicación $(79 \%)$, sociología (80\%) y trabajo social (78\%); debemos rescatar que en las tres escuelas estos porcentajes sobrepasan el $60 \%$ lo que nos lleva a la conclusión que poseen un mejor nivel de rendimiento académico y un eficiente aprendizaje de sus materias.

En la tabla 1 y la figura 1 se puede apreciar que el rendimiento académico a partir del desconocimiento y la no aplicación del aprendizaje colaborativo en las tres escuelas profesionales es regular y malo, observándose los siguientes porcentajes: ciencias de la comunicación (69\%), sociología (60\%) y trabajo social (55\%); debemos rescatar que en las tres escuelas estos porcentajes sobrepasan el $50 \%$ lo que nos lleva a la conclusión que poseen un bajo nivel de rendimiento académico y un deficiente aprendizaje de sus materias.

Por lo tanto la aplicación de estrategias de aprendizaje en las asignaturas por parte de los docentes y el uso de las mismas por parte de los estudiantes en sus materias ha permitido una influencia significativa en el rendimiento académico de los alumnos siendo similar a la propuesta de los hermanos Roger y David Johnson, quienes durante en más de tres décadas se han dedicado a la investigación e implementación de actividades colaborativas desde niveles básicos hasta educación superior. Ellos sostienen que el aprendizaje colaborativo es la base para cualquier técnica que implique trabajo en equipo; va más allá del beneficio individual, ya que no sólo potencia el aprendizaje individual, sino que busca el beneficio del grupo.

En el aprendizaje colaborativo se resalta la importancia de alcanzar objetivos comunes vs. objetivos individuales o de competencia, por lo que ayuda a lograr paralelamente objetivos de aprendizaje y objetivos de habilidades sociales y colaborativas tales como: cooperación, tolerancia, comunicación, escucha activa y compromiso. El trabajo se realiza en grupos pequeños, generalmente heterogéneos, de tres a cuatro integrantes.

Así mismo Johnson, D.R. y Halubec, E. sostienen que el aprendizaje colaborativo es un elemento al que forzosamente tenemos que referirnos cuando hablamos de innovación educativa. Su inserción en el campo educativo ha generado una revolución que mueve el corazón de la práctica docente, la cual hasta hace muy poco había sido dominada por esquemas individuales y de competencia que limitaban los procesos de aprendizaje.

La investigación educativa no sólo ha mostrado el éxito de las prácticas colaborativas en el desarrollo de los estudiantes, sino que ha enfatizado la importancia del desarrollo de habilidades colaborativas como un elemento deseable tanto en la sociedad en la que vivimos como en la sociedad del futuro. (Johnson, D.R. y Halubec, E., 1995).

\section{Conclusiones}

El aprendizaje individual y grupal con la aplicación del aprendizaje colaborativo como estrategia pedagógica docente desarrolla una buena interacción entre estudiantes y docentes; del mismo modo se pudo comprobar que el nivel de aprendizaje en los estudiantes es mejorado y su rendimiento académico también.

Con la aplicación del presente trabajo de investigación se ha logrado revertir los resultados deficientes encontrados en el pre-test, obteniendo en el post-test un incremento significativo en la influencia del rendimiento académico de los estudiantes de la Facultad de Ciencias Sociales; que se muestra en la tabla 3 y la figura 3 donde se aprecia los siguientes porcentajes: ciencias de la comunicación $(79 \%)$, sociología $(80 \%)$ y trabajo social (78\%); debemos rescatar que en las tres escuelas estos porcentajes sobrepasan el $60 \%$ lo que nos lleva a la conclusión que poseen un mejor nivel de rendimiento académico y un eficiente aprendizaje de sus materias.

\section{REFERENCIAS BIBLIOGRÁFICAS}

Canfux, V. (1995). Desarrollo de la Habilidad Profesional de Modelación Estadística para el caso particular de los experimentos comparativos. La HabanaCuba.

Colectivo de autores. (1984). Pedagogía. La HabanaCuba: Pueblo y Educación.

Colectivo de autores. (1995). Los Métodos Participativos. La Habana-Cuba: Universidad de la Habana.

Colectivo de autores. (1996). Tendencias Pedagógicas Contemporáneas. La Habana-Cuba: El Poira.

Díaz, F. y Hernández, G. (2000). Estrategias docentes para un aprendizaje significativo. BogotáColombia: Mc. Graw-Hill.

Hernández, F. (2001). Metodología del Estudio. BogotáColombia: Mc. Graw-Hill.

Talizina, F. (1985). Conferencias sobre los fundamentos de la enseñanza en la educación superior. La Habana-Cuba: Universidad de la Habana.

Viñas, G. (1996). Los métodos participativos. Tesis de maestría. La Habana-Cuba: Universidad de la Habana. 